\title{
Wo kommt das Wasser her? Tracerbasierte Analysen im Rofental (Ötztaler Alpen, Österreich)
}

\author{
Jan Schmieder · Thomas Marke · Ulrich Strasser
}

Online publiziert: 2. Juli 2018

(c) Der/die Autor(en) 2018

\begin{abstract}
Zusammenfassung In diesem Beitrag werden aktuelle tracerhydrologische Forschungsarbeiten an der Rofenache dargelegt, deren hochalpines Einzugsgebiet in den hinteren Ötztaler Alpen liegt. Es wurden Wasserproben von Abfluss, flachgründigen Quellen, Regen, Schnee und Gletscher genommen und auf ihre Sauerstoff-18-Signatur und elektrische Leitfähigkeit analysiert. Die Variabilität der Tracer im Abfluss unterliegt einem deutlichen Jahres- und Tagesgang (letzteres vor allem während der Ablationsperiode). Mit einem tracerbasierten Mischungsmodell konnte ein über den Verlauf der Schneeschmelzperiode variabler Anteil des Schnees am Gesamtabfluss von $35 \%$ im April bis etwa $80 \%$ im Juni abgeschätzt

springs, rain, snow and glaciers were analysed for electrical conductivity and oxygen-18. The streamflow tracer variability shows a marked annual and diurnal cycle, especially during the ablation period. By means of a tracer-based mixing model, both, high snowmelt (up to $80 \%$ ) and glacier melt (up to $75 \%$ ) contributions to streamflow could be attested. The streamwater is dominated by water older than three months, since only $16 \%$ young water fraction was estimated. This could be explained by high fractions of snow and glacier melt, but also occurs due to the deep percolation-typical for some mountainous catchments-which leads to a bypass and contribution to streamflow further downstream.
\end{abstract} werden. Gletscherschmelzanteile konnten mit bis zu $75 \%$ berechnet werden. Das Wasser der Rofenache ist vor allem durch einen geringen Anteil (16\%) an Wasser, welches weniger als drei Monate zuvor als Niederschlag eingetragen wurde, gekennzeichnet. Dies kann durch die hohen Schnee- und Gletscherschmelzanteile, aber auch durch die Tiefensickerung erklärt werden, welche bedingt, dass der eingetragene Niederschlag erst weiter flussabwärts in den Vorfluter gelangt.

Where does the water come from? Tracer-based analyses in the Rofental (Oetztal Alps, Austria)

Abstract This contribution describes recent research in the tracer hydrology of the Rofenache, the mountain stream draining a high-elevation catchment in the European Alps (Oetztal Alps, Austria). Water samples of streamflow,

J. Schmieder, M.Sc. $(\bowtie)$.

Ass.-Prof. Dr. T. Marke .

Univ.-Prof. Dr. U. Strasser

Institut für Geographie, Universität

Innsbruck, Innrain 52f,

6020 Innsbruck, Österreich

jan.schmieder@uibk.ac.at

\section{Hintergrund}

Alpine Einzugsgebiete sind für die Wasserversorgung von Gebirgsregionen und deren Vorländern von besonderer Bedeutung. So ist rund ein Sechstel der Weltbevölkerung vom Wasser aus Gebirgsräumen abhängig (Barnett et al. 2005). Der Wasserüberschuss im Gebirge kann zum einen auf die höheren Niederschläge zurückgeführt werden (der Niederschlag nimmt mit der Höhe zu), von Schnee und Eis, die zeitverzögert abflusswirksam werden und zur hohen Wasserverfügbarkeit beitragen.

In weiten Teilen der europäischen Alpen wird das in kleineren Gerinnen und größeren Flüssen abfließende Wasser zumeist für die Energieerzeugung durch Wasserkraft sowie für die Bewässerung landwirtschaftlicher Nutzflächen eingesetzt (Schaefli et al. 2007; Beniston 2012). Aber auch der Tourismus ist vom Wasser abhängig - so haben die Hotels im Vergleich zur lokalen Bevölkerung durch die hohen Nächtigungszahlen einen großen Anteil am regionalen Wasserverbrauch. Zudem erfordert in Wintersportdestinationen auch die Produktion von technischem Schnee einen hohen Waszum anderen sind es die Schmelzwässer sereinsatz (Hanzer et al. 2014; Rixen et al. 2011).

In hochalpinen Einzugsgebieten wird das Wasserdargebot zu einem großen Anteil durch langfristig (Gletscher) bzw. saisonal (Schnee) gespeicherte Wasserressourcen und deren Schmelze während der Frühjahrs- und Sommermonate gesteuert. Im Gerinne sind also Wasser von unterschiedlichem Alter und Herkunft, mit tageszeitlich, saisonal und durch den Klimawandel auch dekadisch sich ändernden Anteilen. Die zahlenmäßige Quantifizierung dieser Anteile ist eine wichtige Aufgabe für die Wasserwirtschaft. Dieser Beitrag soll dazu einen Beitrag liefern; er gibt einen Überblick über die aktuellen tracerhydrologischen Forschungsarbeiten im Rofental (Tirol, Österreich), mit besonderem Fokus auf der Untersuchung des Abflussverhaltens, der Abflusszusammensetzung und der Tracerreaktion der Rofenache.

\section{Einführung}

Trotz der langjährigen Forschung im Rofental, welche stimuliert durch die internationale hydrologische Dekade ab 1965, initiiert von der UNESCO (Hoinkes et al. 1974) weiter intensiviert wurde, bleiben viele Fragen zur Abflussbildung und zur Herkunft des Wassers in Gebirgsregionen unbeantwortet. Durch Einsatz tracerhydrologischer Methoden ist es möglich, die Anteile aus Gletscher- und Schneeschmelze, Niederschlag und Grundwasser $\mathrm{zu}$ trennen und $\mathrm{zu}$ quantifizieren. Aufgrund der vielseitigen Untersuchungsmöglichkeiten finden Tracer (Markierungsmittel) in der Hydrologie bereits seit den 1960er-Jahren großen Einsatz (Klaus und McDonnell 2013). Ideale Tracer verhalten sich wie Wassermoleküle selbst und ändern ihre Signatur nicht durch sich verändernde Umweltbedingungen (z. B. beim Durchfließen von Grundwasserspeichern). Durch das Gesetz der Massenerhaltung kann bei Mischung von Wasser mit un- 


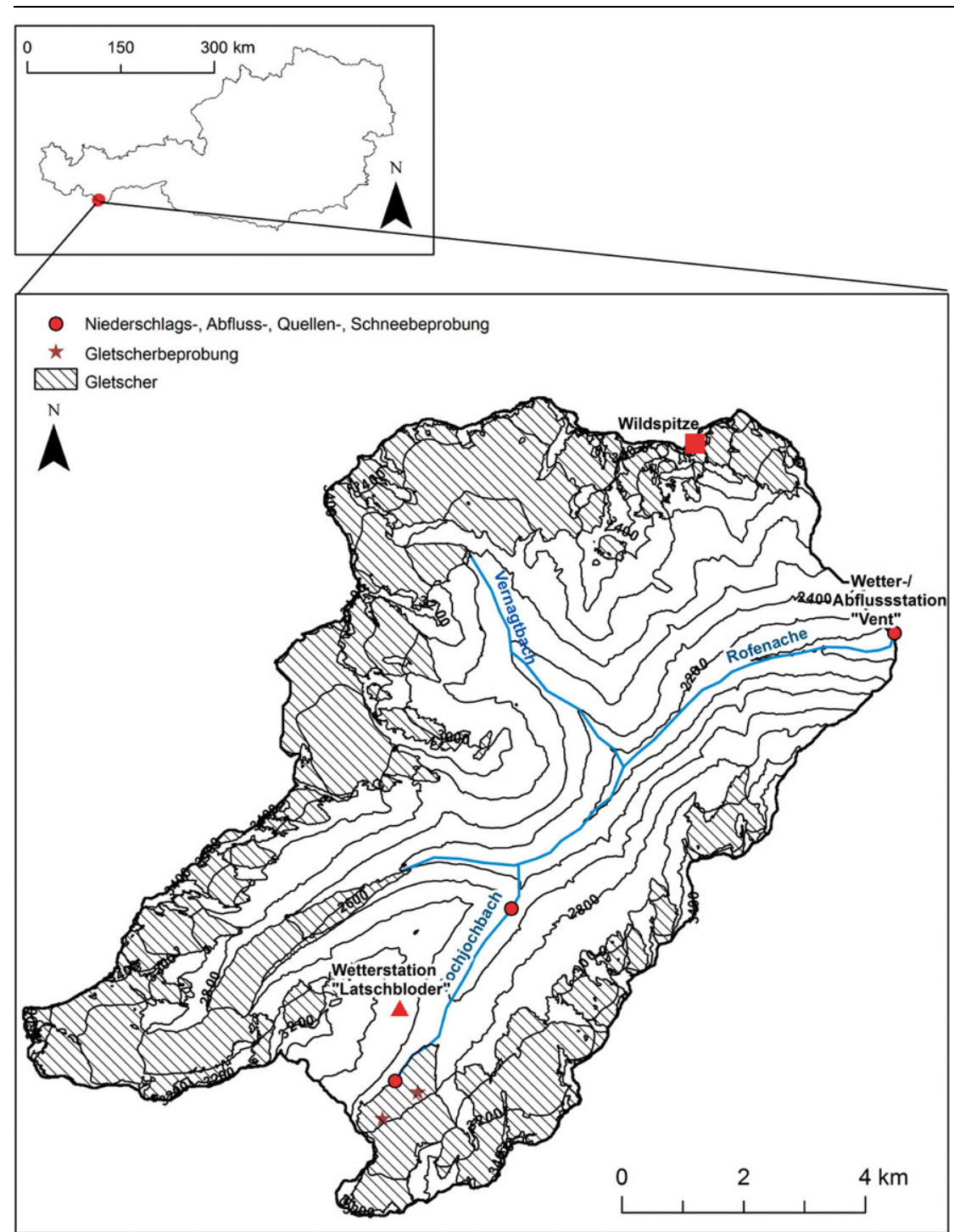

Abb. 1 Einzugsgebiet der Rofenache in den Ötztaler Alpen, Österreich

terschiedlichen Tracersignaturen unter Verwendung sogenannter „Mischungsmodelle" auf die jeweiligen Anteile aus unterschiedlichen Herkunftsräumen geschlossen werden. Oft finden als natürliche Tracer (auch „Umwelttracer") die Gehalte an stabilen Isotopen des Wassers (z. B. Sauerstoff-18 $\left(\delta^{18} \mathrm{O}\right)$ ) Betrachtung, wobei neben der Bestimmung von Verweilzeiten und Herkunftsräumen des Wassers auch auf vorherrschende Abflussbildungsmechanismen geschlossen werden kann. Mit Hilfe von isotopischen Tracern kann dabei durch einen Vergleich von saisonalen Inputtracerzyklen (Niederschlag) und Outputtracerzyklen (Abfluss) die mittlere Verweilzeit und der Anteil an „young water" (Wasser welches weniger als drei Monate im Einzugsgebiet verweilt) abgeschätzt werden (Kirch-
Wasser herabfallender Tropfen), verstärkt jedoch nach dem Auftreffen im Einzugsgebiet durch alle Arten von Phasenübergängen $\mathrm{zu}$ einer Veränderung der Isotopensignatur. Dabei gehen die leichteren Sauerstoff-16-Isotope beim Phasenübergang im Vergleich zu den schwereren Sauerstoff18-Isotopen leichter in den energetisch höheren Zustand über (z.B. bei Verdunstung). In umgekehrter Weise lässt sich dieser Effekt beim Übergang in die energetisch niedrigere Phase beobachten (z.B. bei Kondensation, Gat 2010). Somit resultieren die Anund Abreicherung von Sauerstoffisotopen in unterschiedlichen Sauerstoff18-Signaturen für Wasserproben aus Niederschlag, Grundwasser, Schnee, Gletscher oder Quellen. Je nach Anzahl der gelösten Ionen steigt die elektrische Leitfähigkeit (LF) dabei an, abhängig von der bereits vergangenen Zeit sowie der gegebenen Löslichkeit der Ionen im Gestein und im Boden. Um die Anteile aus Niederschlag, Schnee- und Eisschmelze und Grundwasser verlässlich trennen zu können, muss die raumzeitliche Variabilität der Signaturen der abflussbeitragenden Komponenten quantifiziert werden. Der Einfluss dieser räumlichen und zeitlichen Variabilität wurde in zwei vorangegangenen Studien bereits für die Rofenache untersucht und dokumentiert (Schmieder et al. 2016, 2018).

Im vorliegenden Beitrag können daran anschließend neben der Erweiterung des zugrunde liegenden Datensatzes (i) die Untersuchung der zeitlichen Tracervariabilität des Abfluss auf Tagesund Jahresskala sowie (ii) die Analyse der Beziehung zwischen Tracersignatur und Abfluss während verschiedener Systemzustände fortgeführt werden.

\section{Das Untersuchungsgebiet Rofental}

Das Rofental ist im hinteren Ötztal (Tirol, Österreich) gelegen und erstreckt sich von $1900 \mathrm{~m}$ (Pegel Vent) bis auf $3770 \mathrm{~m}$ (Gipfel der Wildspitze) mit einer mittleren Einzugsgebietshöhe von $2950 \mathrm{~m}$ (siehe Abb. 1; Strasser et al. 2018). Das langjährige Jahresmittel der Temperatur beläuft sich auf $2^{\circ} \mathrm{C}$, das langjährige Jahresmittel des Niederschlags beträgt $800 \mathrm{~mm}$ (Werte der Station Vent, 1982-2003). Geologisch ist das Gebiet durch kristallines Gestein geprägt, wobei hauptsächlich Paragneis und Glimmerschiefer vorkommen. Das 


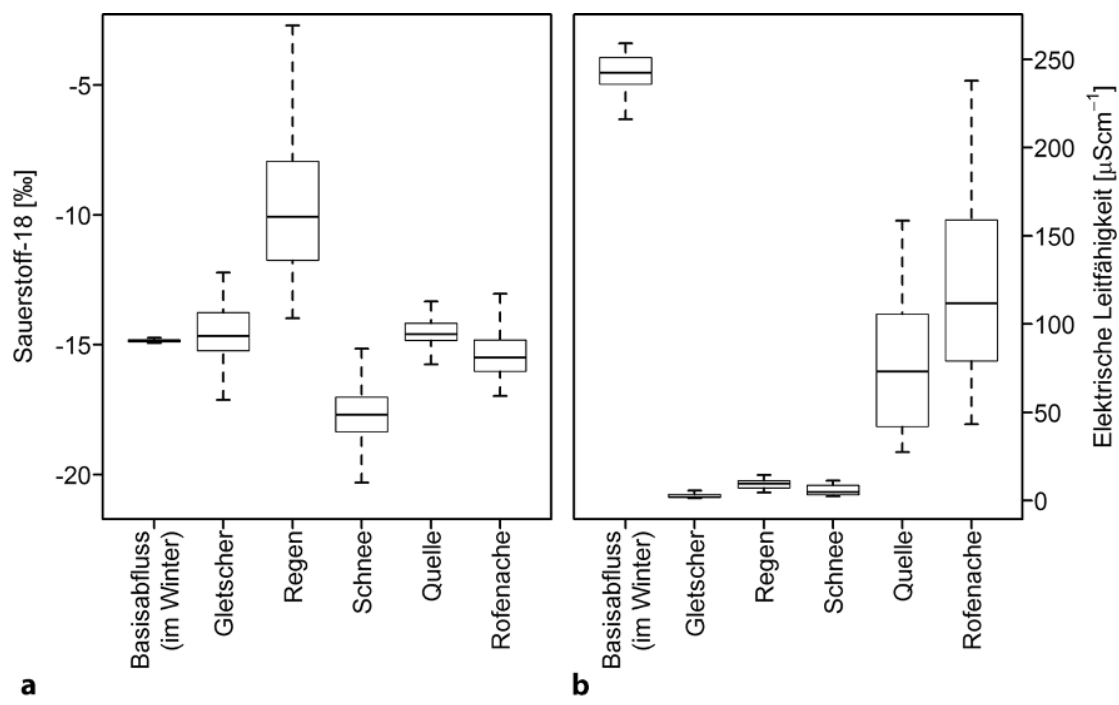

Abb. 2 Sauerstoff-18-Signatur (a) und elektrische Leitfähigkeit (b) von Wasserproben verschiedener Herkunftsräume im Rofental (579 Proben aus den Jahren 2014-2017)

Gebiet ist zu ca. $42 \%$ von Lockermaterial und Grundgestein bedeckt und kaum bewaldet $(<1 \%)$. Rund ein Drittel der Gesamtfläche von $98 \mathrm{~km}^{2}$ wird derzeit von Gletschern bedeckt (Fischer et al. 2015). Der Ursprung der Rofenache ist das Gletschertor des Hintereisferners, wobei die Rofenache in ihrem Verlauf durch zwei größere Fließgewässer erster Ordnung (Hochjochbach und Vernagtbach) sowie weitere kleine Fließgewässer gespeist wird. Die Rofenache weist ein glaziales Abflussregime auf, mit großer Abflussvariabilität zur Zeit des Abflussmaximums im Sommer und geringer Variabilität zur Zeit des Abflussminimums im Winter. Der mittlere Jahresabfluss beträgt $4,5 \mathrm{~m}^{3} / \mathrm{s}$ (1971-2009). Da die Rofenache nicht für die Energiegewinnung aus Wasserkraft genutzt wird, kann ihr Zustand als sehr naturnah beschrieben werden. Dieser Umstand macht das Einzugsgebiet der Rofenache zum idealen Hochgebirgslabor für die hydrologische Prozessforschung.

\section{Methoden}

Die in dieser Studie analysierten Abflussdaten vom Pegel in Vent wurden vom hydrografischen Dienst Tirol bezogen. Die Wasserproben wurden im Untersuchungsgebiet mithilfe studentischer Mitarbeiter/innen gesammelt. Die zugehörigen Standorte der Probenentnahme sind in die Karte des Untersuchungsgebiets eingetragen (Abb. 1). Die elektrische Leitfähigkeit (in $\mu \mathrm{S} / \mathrm{cm}$ ) als Summenparameterindex für die
Masse an gelösten Stoffen im Wasser wird im Gelände mit einem tragbaren Taschengerät bestimmt. Die Wasserproben von Abfluss, flachgründigen Quellen, Regen, Schnee und Gletscher wurden im Labor des Instituts für Meteorologie und Klimaforschung (Atmosphärische Umweltforschung) in Garmisch-Partenkirchen, einer Außenstelle des Karlsruher Instituts für Technologie, sowie im Isotopenlabor des Geologischen Instituts der Universität Bern analysiert. Die Laborergebnisse umfassen die Sauerstoff-18-Isotopensignatur (in \%o) und deren Laborgenauigkeit.

Die tracerbasierte Abflusskomponententrennung (auch Mischungsmodell) wurde von Pinder und Jones (1969) entwickelt und beruht auf dem physikalischen Massenerhaltungsgesetz. Bei unterschiedlichen Tracersignaturen) kann damit auf die prozentualen Wasseranteile der Mischung geschlossen werden. Ein entscheidender Durchbruch im hydrologischen Prozessverständnis humid-gemäßigter Einzugsgebiete gelang durch den Einsatz von Tracern, indem nachgewiesen wurde, dass der Abfluss großteils aus Anteilen besteht, welche schon vor dem Regenereignis im System gespeichert waren (Klaus und McDonnell 2013). Dies ist in Regionen mit saisonaler Schneedecke oder Vergletscherung jedoch seltener zu beobachten, und viele Forschungsfragen dazu bleiben ungeklärt. Vor allem die schwierige Zugänglichkeit der Hochgebirge verhindert hier ein rasches Voranschreiten von ForschungsarbeiMischung von Stoffen (z. B. Wasser mit ten. Grundvoraussetzung für die erfolgreiche Anwendung eines Mischungsmodells sind signifikante Unterschiede in den Tracersignaturen der Abflusskomponenten. Eine Übersicht für die Anwendung der Mischungsmodelle ist in Klaus und McDonnell (2013) angeführt. Die Unsicherheit dieser Methode wird häufig mit dem Ansatz von Genereux (1998) abgeschätzt, welche auf der Gauß'schen Fehlerfortpflanzung beruht. Die Laborgenauigkeit, die raumzeitliche Variabilität, sowie der Unterschied in der Tracerzusammensetzung der abflussbeitragenden Komponenten steuern hierbei die Größenordnung der Unsicherheit.

\section{Erkenntnisse}

\subsection{Tracervariabiltät}

\subsubsection{Die Tracersignatur der Herkunftswasser und des Abflusses}

Abb. 2 zeigt die Verteilung der Tracersignatur von winterlichen Basisabfluss-, Regen-, Schnee-, Gletscher-, Quell- und Abflussproben aus dem Einzugsgebiet der Rofenache für Sauerstoff-18 (Abb. 2a) und elektrische Leitfähigkeit (LF; Abb. 2b) für den Zeitraum März 2014 bis Dezember $2017(n=579)$. Regen weist höhere und Schnee vergleichsweise geringere Sauerstoff-18Werte auf, eng verknüpft mit der Temperatur bei der Niederschlagsbildung. Gletscher, Schnee und Regen haben sehr niedrige LF-Werte $(<10 \mu \mathrm{S} / \mathrm{cm})$, bedingt durch den fehlenden Kontakt mit dem Untergrund und der dadurch unterbundenen Aufnahme von Ionen. Die LF wird in diesem Fall durch die atmosphärische Deposition sowie durch die sukzessive Auswaschung der Ionen während der Schmelze von Schnee und Eis bestimmt. Der winterliche Basisabfluss zeichnet sich durch hohe LFWerte aus, was ein Indiz für Wasser mit längeren Verweilzeiten ist, bei dem die Möglichkeit der Ionenlösung vom durchflossenen Medium gegeben ist. Die beprobten flachgründigen Quellen weisen hingegen deutlich niedrigere LF-Werte auf, ein Hinweis auf kürzere Verweilzeiten unter Annahme gleicher Löslichkeit der Ionen. Deutlich erkennbar sind sowohl große Spannweiten in der Sauerstoff-18-Signatur von Gletscher-, Regen- und Schneeproben, als auch eine markante Streuung in der elektrischen Leitfähigkeit in Was- 


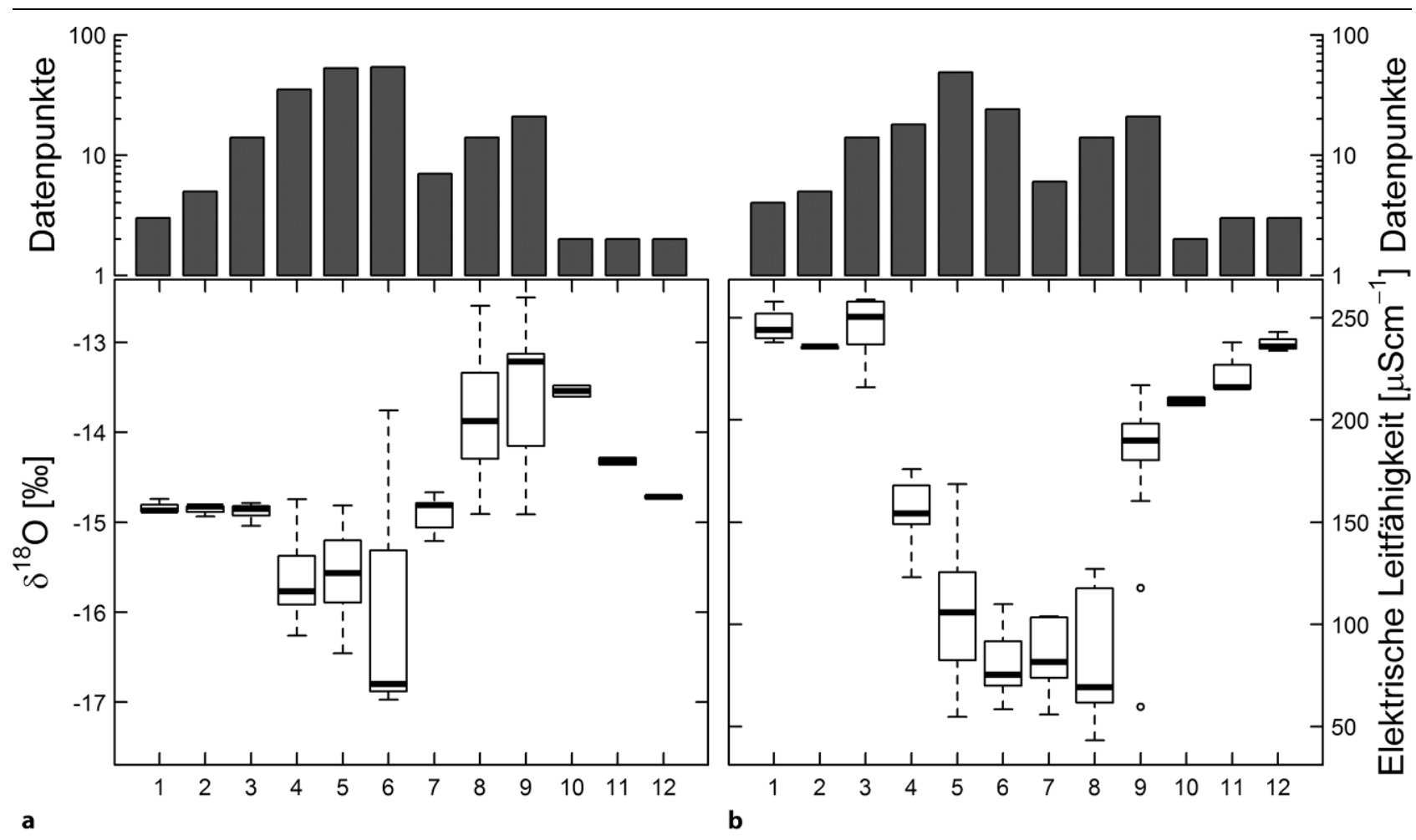

Abb. 3 Monatliche Tracersignatur der Rofenache für $\delta^{18} \mathrm{O}(\mathbf{a})$ und elektrische Leitfähigkeit (b). Die analysierten Wasserproben wurden zwischen 2014 und 2017 erhoben

serproben aus flachgründigen Quellen. Signifikante Unterschiede in der Sauerstoff-18-Signatur von Gletscher-, Schnee- und Regenproben sind eine Voraussetzung für die Anwendbarkeit des tracerbasierten Mischungsmodells (vgl. Methodenteil). Da sich der winterliche Basisabfluss diesbezüglich nicht signifikant von den Gletscherproben unterscheidet (vgl. Abb. 2a), ist hier ein weiterer Tracer unabdingbar. Die elektrische Leitfähigkeit als weiterer und herkunftsraumorientierter Tracer für das Mischungsmodell ist hierbei hilfreich, da sich die Werte des Basisabflusses darin signifikant von denen der Regen-, Schnee- und Gletscherproben unterscheiden (vgl. Abb. 2b).

\subsubsection{Der Jahresgang der Tracersignatur im Abfluss}

Die Tracersignatur von Hochgebirgsflüssen unterliegt häufig einem sinusartigen Jahresgang, verursacht durch hydrologische Prozesse, welche einen ausgeprägten Jahresgang aufweisen (z.B. Energieinput für Schmelzprozesse, abflusswirksame konvektive Regenereignisse). Bisher konnten am Pegel Vent/Rofenache 216 Proben genommen und analysiert werden. Auffällig ist hierbei der markante Tiefpunkt zur Zeit der Ablationsperiode $\left(\delta^{18} \mathrm{O}\right.$ : Juni, LF: August). Abb. 3 zeigt die monatliche und jährliche Variation der $\delta^{18} \mathrm{O}$ (Abb. 3a) und LF-Werte (Abb. 3b) für den Zeitraum 2014 bis 2017. Typisch dabei ist die durch die abflussbeitragenden Komponenten gesteuerte geringe Variation der $\delta^{18} \mathrm{O}$ - und LF-Werte während der Wintermonate (Dezember bis März) sowie deren hohe Variabilität während der Ablationsperiode (April bis September). Ein besonderes Merkmal vergletscherter Einzugsgebiete (mit über $30 \%$ Gletscherflächenanteil) ist die Korrelation der beiden analysierten Tracer. Von April bis Juni korrelieren LF und $\delta^{18} \mathrm{O}$ des Abflusses positiv miteinander. Im Juli wird diese Korrelation unterbrochen, da die $\delta^{18} \mathrm{O}$-Werte nun wieder ansteigen, während die LF-Werte den jährlichen Tiefpunkt erreichen. Dieser Zeitpunkt markiert im Jahresverlauf den Einsatz der Eisschmelze und damit den Übergang von schneeschmelzdominierter Abflussbildung zu gletscherschmelzdominierter Abflussbildung. Diese Hypothese wird gestützt durch die relativ hohen $\delta^{18} \mathrm{O}$ - und sehr geringen LF-Werte der Gletscherschmelzwasserproben im Vergleich zu den Schneeproben, sollte aber durch die bisherige Einzigartigkeit dieses Datensatzes in anderen vergletscherten Einzugsgebieten getestet werden.

\subsubsection{Die Beziehung zwischen Tracersignatur und Abfluss}

Die Tracersignatur von Hochgebirgsflüssen unterliegt während der schneeund eisschmelzdominierten Ablationsperiode häufig auch einem ausgeprägten Tagesgang, welcher durch den Tagesgang der Schmelzprozesse gesteuert wird. Abb. 4 zeigt die Beziehung der Tracersignatur zum Abfluss während eines Tages (2-h-Intervall; 16. bis 17. Mai 2017). Das Beispiel zeigt eine rechtsläufige Hysterese für beide Tracer $\left(\delta^{18} \mathrm{O}\right.$ und LF). Während des ansteigenden Astes der Abflussganglinie treten bei gleichem Abfluss höhere Tracersignaturen auf als während des absteigenden Astes. Die Form der Hysterese der beiden Tracer unterscheidet sich deutlich, was auf ein komplexes Zusammenspiel mehrerer der bedingenden Faktoren schließen lässt. Der Schneeschmelzanteil nimmt dabei von etwa $30 \%$ auf über $60 \%$ stark zu. Die beobachtete Hysterese kann durch das dynamische Auftreten und Wirken der verschiedenen Abflusskomponenten 


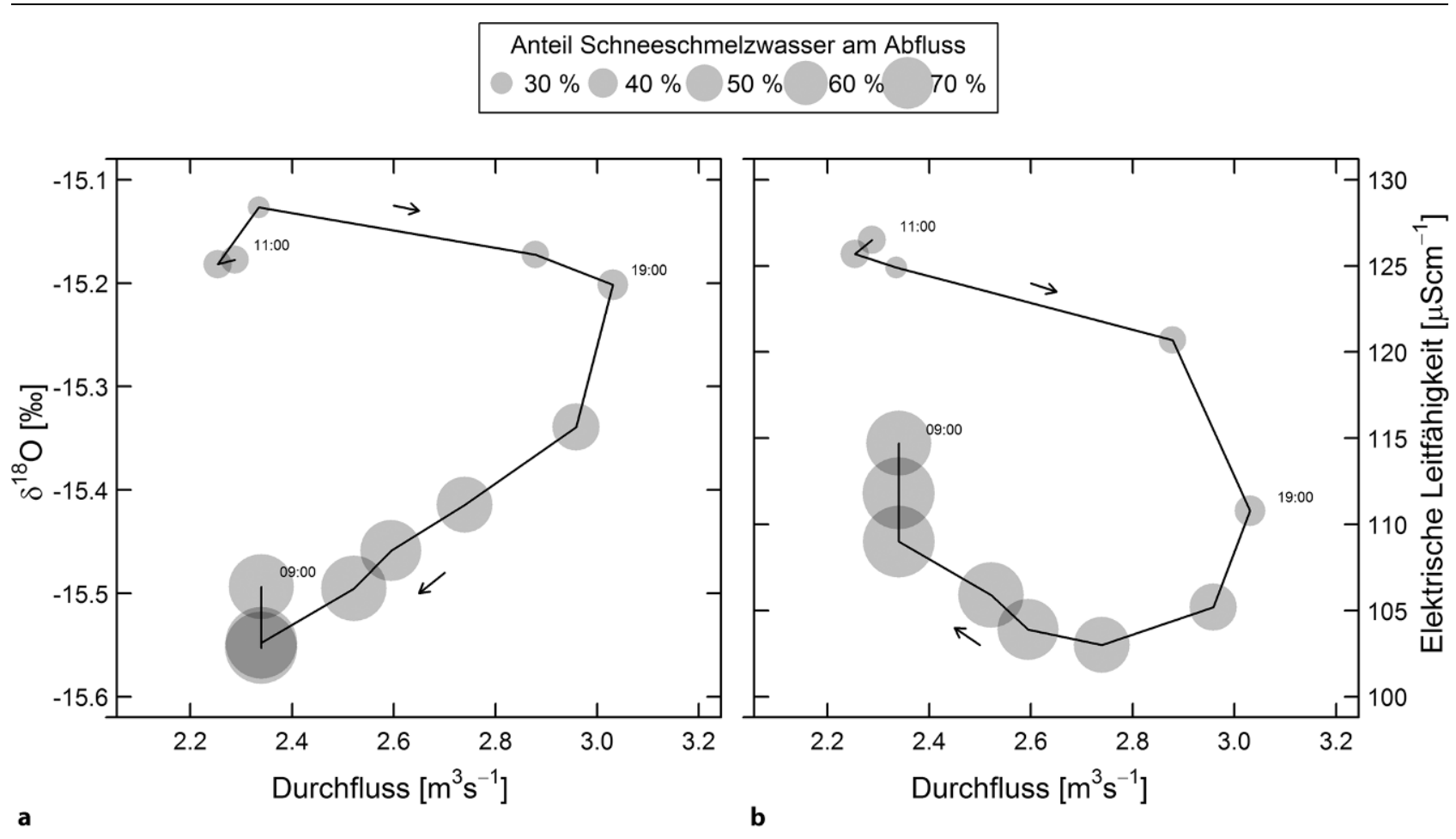

Abb. 4 Die Beziehung zwischen der Tracersignatur und des Abflusses der Rofenache zeigt für den typischen Tagesgang eines Schneeschmelzereignisses eine rechtsläufige Hysterese

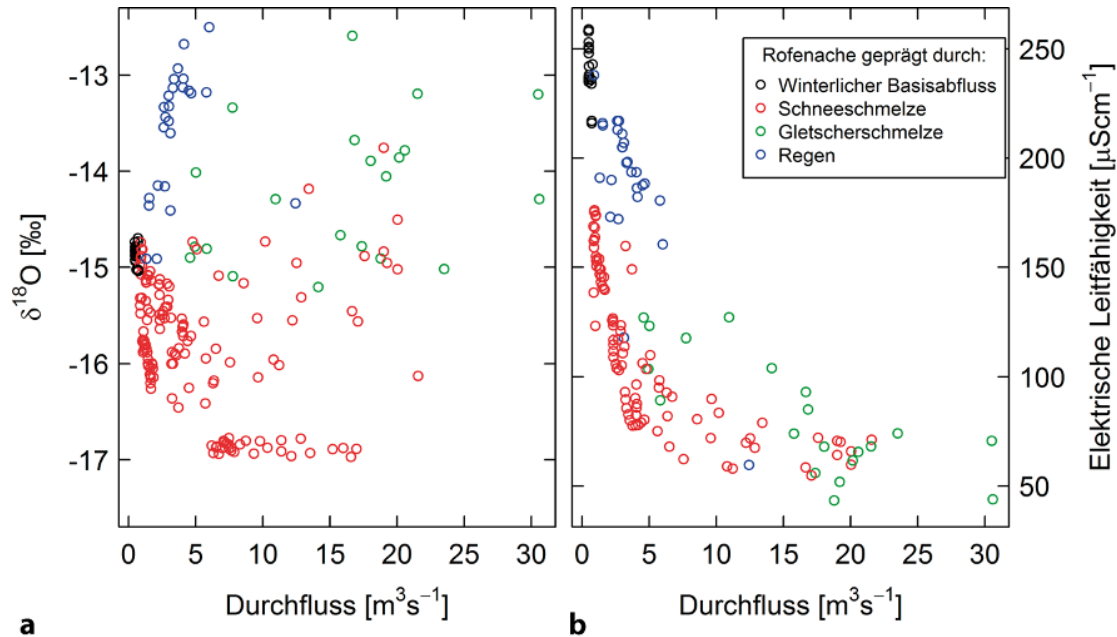

Abb. 5 Beziehung zwischen der Tracersignatur der Rofenache und deren Durchfluss für $\delta^{18} \mathrm{O}$ (a) und die elektrische Leitfähigkeit (b). Die analysierten Wasserproben wurden zwischen 2014 und 2017 erhoben

entstehen (Evans und Davies 1998), wie beispielsweise auch von Engel et al. (2016) in einem vergleichbaren Untersuchungsgebiet beobachtet.

Abb. 5 zeigt die Tracersignatur und den zugehörigen Abfluss der Rofenache zu jedem Zeitpunkt der Probenentnahme für die vierjährige Untersuchungsperiode. Vor allem für die LF zeigt sich hier ein erkennbarer Zusammenhang, der häufig mit dem positiven Ast einer Hyperbel beschrieben wird (Hellmann 1999). Die Einfärbung der Datenpunkte wurde nach der Klassifikation von dominierenden Phasen vorgenommen (winterlicher Basisabfluss: Dezember bis März; Schneeschmelze: April bis Juni, Gletscherschmelze: Juli bis August; Regen: September bis November) und zeigt Cluster für beide Tracer. Die Daten sind charakterisiert durch tendenziell höhere Tracersignaturen bei geringen Abflüssen bis hin zu eher geringeren Tracerwerte bei höheren Abflüssen, wobei die $\delta^{18} \mathrm{O}$-Werte deutlich mehr Streuung aufweisen (vgl. Abb. 5a).

\subsection{Abflusszusammensetzung der Rofenache}

\subsubsection{Schneeschmelzbeitrag zum Gesamtabfluss}

Für die Schneeschmelzperiode 2014 konnten in einer ereignisbasierten Studie (Schmieder et al. 2016) die Schneeschmelzanteile mittels eines Zwei-Komponenten-Mischungsmodells für April und Juni abgeschätzt werden, wobei sich die Systemzustände zwischen beiden Ereignissen deutlich unterschieden. Der verwendete Tracer war $\delta^{18} \mathrm{O}$. Im April lag der geschätzte Anteil des Schneeschmelzwassers im Abfluss bei $35 \%$ (Abb. 6a). Der Abfluss wurde dominiert durch Wasser, welches schon vorher im Einzugsgebiet gespeichert war und dann erst durch die Schneeschmelze abflusswirksam wurde. Während der Hauptschneeschmelzperiode im Juni (Abb. 6b) wurden maximale Schneeschmelzwasseranteile im Abfluss von bis zu $80 \%$ erreicht. Der Abfluss wurde 


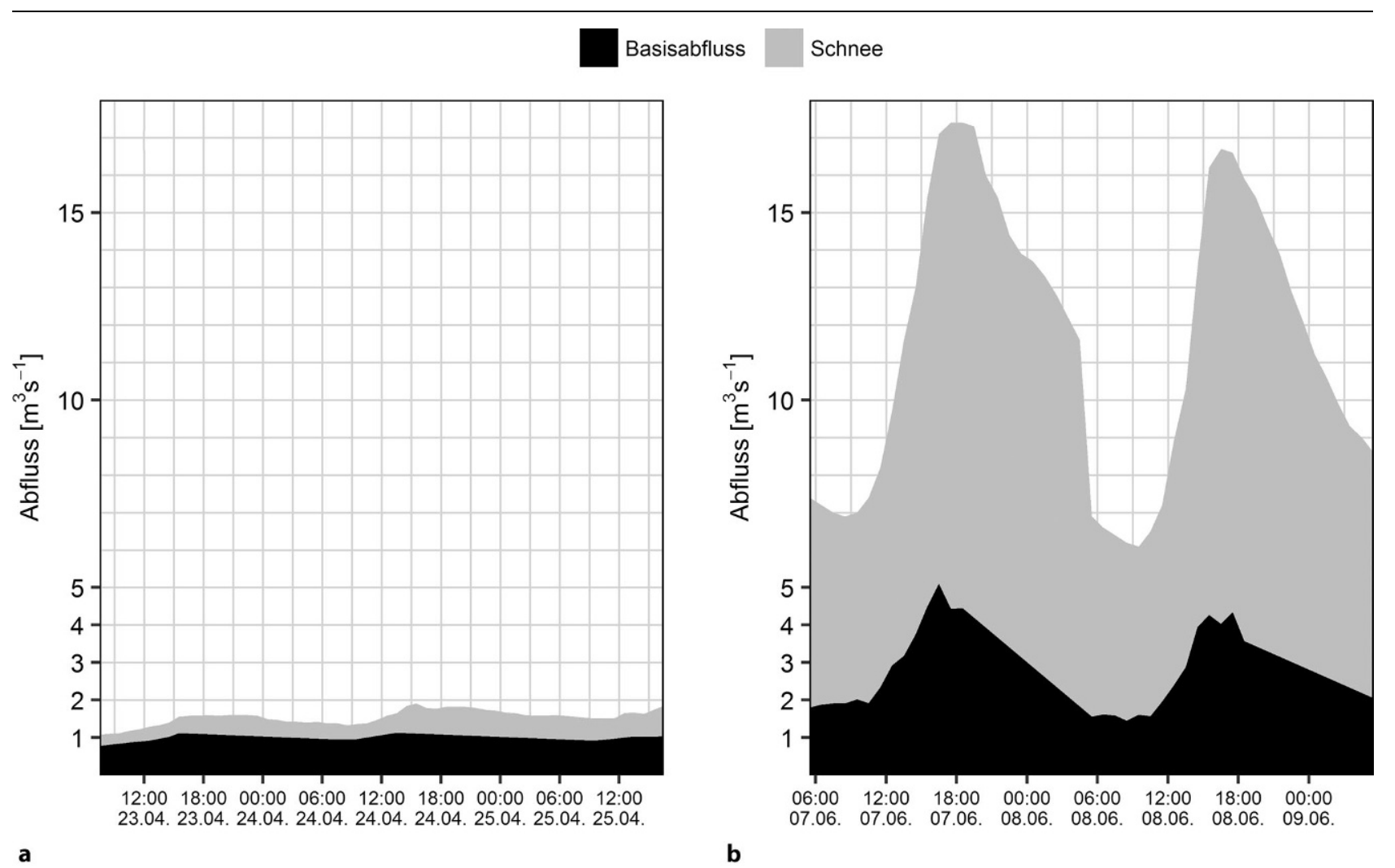

Abb. 6 Abflussanteile der Rofenache während eines frühen Schneeschmelzereignisses (a) und eines Ereignisses in der Hauptschneeschmelzperiode (b)

Regen $\square$ Grundwasser $\square$ Gletscher

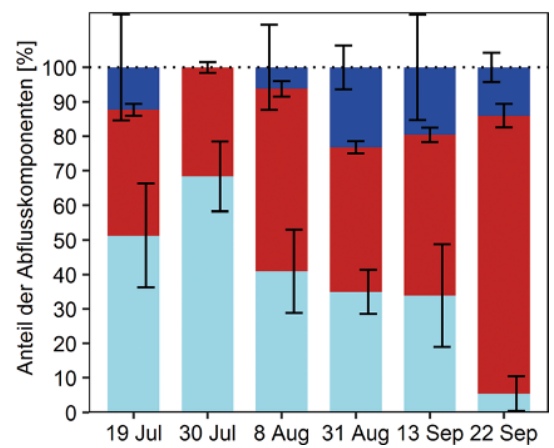

Abb. 7 Abflussanteile des Hochjochbaches während sechs Tagen in der Ablationsperiode 2016 des Hochjochferners. Die Fehlerbalken zeigen die Unsicherheit an (mit $95 \%$ Konfidenz)

dabei eindeutig von Wasser dominiert, welches dem System durch die Schneeschmelze hinzugefügt wurde.

\subsubsection{Gletscherschmelzbeitrag zum Gesamtabfluss}

Für die Gletscherschmelzperiode 2016 konnten in einer weiteren ereignisbasierten Studie (Schmieder et al. 2018) die Gletscherschmelz-, Regen- und Grundwasseranteile im Abfluss abgeschätzt werde. Dazu wurde ein DreiKomponenten-Mischungsmodell $\left(\delta^{18} \mathrm{O}\right.$ und LF) für sechs Ereignisse in einem Teileinzugsgebiet der Rofenache (Hochjochbach, $17 \mathrm{~km}^{2}, 34 \%$ Vergletscherung) angewandt. Hier konnten maximale Gletscherschmelzwasseranteile im Abfluss von bis zu etwa $75 \%$ aufgezeigt werden (Abb. 7), wobei der Grundwasseranteil während dieser sechs Ereignisse dominant war (49\%). Dabei bleibt festzuhalten, dass die sechs Ereignisse Stichproben während der Ablationsperiode darstellen und weitere Daten notwendig wären, um z.B. auf Gletschermassenverluste schließen zu können.

\subsection{Der Anteil von „young water" im Abfluss der Rofenache}

Über die Zusammensetzung des Wassers hinaus kann mit stabilen Isotopen auch das Alter des Wassers bestimmt werden. Eine neue Methode wurde durch Kirchner (2016a, 2016b) entwickelt, mit welcher der Anteil an „young water“ abgeschätzt werden kann. „Young water“ ist per Definition nicht älter als drei Monate, das heißt es verweilt weniger als drei Monate im Einzugsgebiet zwischen Eintrag (als Niederschlagsereignis) und dessen Austrag durch den Abfluss des Vorfluters. Für die Anwendung der Methode benötigt es dazu monatliche Isotopendaten aus Niederschlag und Abfluss für mindestens ein Jahr. Diese werden mit einer trigonometrischen Funktion durch multiple lineare Regression angepasst und aus dem Quotient der Amplitude des Abflusses und der des Niederschlags wird der Anteil des „young water“ berechnet. Der mittlere Anteil an „young water“ der Rofenache während der Jahre (1972-1977 und 2015-2016) betrug 16\% (Standardabweichung: 11\%; Maier 2017). Jasechko et al. (2016) untersuchten in einer globalen Studie die Anteile an „young water" auf globaler Skala und berechneten sehr geringe Werte von meist unter $10 \%$ für Hochgebirgsflüsse. Als Grund dafür dient die Hypothese, dass einerseits durch die zeitverzögernde Zwischenspeicherung des Niederschlages im Schnee (und Gletscher) der Anteil geringer ist als im Tiefland, und 
dass andererseits die Tiefenversickerung durch das Grundgestein in gebirgigen Einzugsgebieten sehr viel höher ist als bisher angenommen. Dadurch gelangt das Wasser der Kopfeinzugsgebiete erst in tiefer gelegenen Regionen in den Vorfluter (Gleeson und Manning 2008). Die Daten der Rofenache lassen sich gut in die globalen Daten einordnen und die oben genannte Hypothese als Begründung dazu erscheint plausibel.

\section{Synthese}

Der vorliegende Beitrag beschreibt die tracerhydrologischen Untersuchungen im Rofental während der Periode 2014 bis 2017. Markante Tagesgänge während der Ablationsperiode, sowie jahreszeitliche, sinuskurvenartige Verläufe konnten für beide Tracer im Abfluss der Rofenache beobachtet werden. Dieses Verhalten konnte mit Mischungsmodellen erklärt werden, welche die Abflusszusammensetzung quantifizieren, und wird durch zeitlich variable Abflussanteile an Schnee, Eis, Regen und Grundwasser gesteuert. Das Wasser der Rofenache ist dabei vorwiegend älter als drei Monate, was auf vorhandene unterirdische Speichersysteme (neben der saisonalen Schneedecke und der Gletscher) in diesem Einzugsgebiet schließen lässt.

\section{Ausblick}

Im Anschluss an die bisher abgeschlossenen ereignisorientierten Studien zur Tracervariabiltät der Wasser verschiedener Herkunftsräume und zum Abflussverhalten in der Ablationsperiode stehen nun Regenereignisse und deren komplexes Zusammenspiel mit Schmelzprozessen im Rofental im Fokus. Dies und auch die Analyse von Grundwasserneubildungs- und Grundwasserabflussprozessen in vergletscherten Einzugsgebieten stellen einen spannenden Forschungsbereich mit internationaler Wahrnehmung dar. In einem weiteren Schritt werden mit dem tracerbasierten Mischungsmodell die Abflusskomponenten auf jährlicher Skala abgeschätzt. Damit soll dann eine dreijährige Zeitreihe von Abflussanteilen mit mindestens monatlichen Zeitschritten entstehen, welche im weiteren Verlauf mit simulierten Abflussanteilen des hydroklimatotologischen Modells AMUNDSEN (Strasser 2008) verglichen wird.

Danksagung Der vorliegende Beitrag ist ein Teil des Forschungsprojektes HydroGeM $^{3}$, welches durch die Österreichische Akademie der Wissenschaften gefördert wird. Das Projekt wird in Kooperation mit dem Institut für Geographie der Universität Bern durchgeführt, welches vergleichbare Studi- en im schweizerischen Einzugsgebiet der Lütschine durchführt. Besonderer Dank gilt unseren Laborpartnern für die Analyse der stabilen Isotope des Wassers, dem Center of Stable Isotopes des Karlsruher Instituts für Tewchnologie, sowie dem stabilen Isotopenlabor des Geologischen Instituts der Universität Bern. Ausgesprochen dankbar sind wir außerdem dem Hydrographischen Dienst Tirol für die Installation des automatischen Probennehmers und für die Bereitstellung der Abflussdaten von der Pegelstation in Vent. Großer Dank gilt auch den studentischen MitarbeiterInnen, ohne die die Geländearbeit in diesem Umfang nicht durchführbar gewesen wäre.

Funding Open access funding provided by University of Innsbruck and Medical University of Innsbruck.

Open Access Dieser Artikel wird unter der Creative Commons Namensnennung 4.0 International Lizenz (http:// creativecommons.org/licenses/by/4. $0 /$ deed.de) veröffentlicht, welche die Nutzung, Vervielfältigung, Bearbeitung, Verbreitung und Wiedergabe in jeglichem Medium und Format erlaubt, sofern Sie den/die ursprünglichen $\mathrm{Au}$ tor(en) und die Quelle ordnungsgemäß nennen, einen Link zur Creative Commons Lizenz beifügen und angeben, ob Änderungen vorgenommen wurden.
Barnett, T. P., Adam, J. C., and Lettenmaier, D. P. (2005): Potential impacts of a warming climate on water availability in snow-dominated regions, Nature, 438, 303-309.

Beniston, M. (2012): Impacts of climatic change on water and associated economic activities in the Swiss Alps, Journal of Hydrology, 412-413, 291-296, https://doi.org/10.1016/j.jhydrol.2010. 06.046 .

Dansgaard, W. (1964): Stable isotopes in precipitation, Tellus, 16, 436-468, https://doi.org/10 1111/j.2153-3490.1964.tb00181.x.

Engel, M., Penna, D., Bertoldi, G., Dell'Agnese, A., Soulsby, C., and Comiti, F. (2016): Identifying run-off contributions during melt-induced runoff events in a glacierized alpine catchment. $\mathrm{Hy}$ drological Processes, 30(3), 343-364. https://doi. org/10.1002/hyp.10577.

Evans, C., and Davies, T.D. (1998): Causes of concentration/discharge hysteresis and its potential as a tool for analysis of episode hydrochemistry. Water Resources Research, 34(1), 129-137. https://doi.org/10.1029/97WR01881.

Fischer, A., Seiser, B., Stocker-Waldhuber, M. Mitterer, C., and Abermann, J. (2015): Tracing glacier changes in Austria from the Little Ice Age to the present using a lidar-based high-resolution glacier inventory in Austria, The Cryosphere,
9, 753-766, https://doi.org/10.5194/tc-9-7532015.

Gat, J.R. (2010): Isotope Hydrology: A Study of the Water Cycle, Imperial College Press.

Gleeson, T., and Manning, A.H. (2008): Regional groundwater flow in mountainous terrain Three-dimensional simulations of topographic and hydrogeologic controls. Water Resources Research, 44(10), n/a-n/a. https://doi.org/10. 1029/2008WR006848.

Hanzer, F., Marke, T., and Strasser, U. (2014): Distributed, explicit modeling of technical snow production for a ski area in the Schladming region (Austrian Alps), Cold Regions Science and Technology, 108, 113-124, https://doi.org/10. 1016/j.coldregions.2014.08.003.

Hellmann, H. (1999): Qualitative Hydologie Wasserbeschaffenheit und Stoff-Flüsse. Stuttgart: Borntraeger.

Hoinkes, H., Dreiseitl, E., and Wagner, H. P. (1974): Mass Balance of Hintereisferner and Kesselwandferner 1963/64 to 1972/73 in Relation to the Climatic Environment, Preliminary results of the cornbined water, ice and heat balances project in the Rofental, IHD-Activities in Austria 1965-1974, Report of the Int. Conference on the Results of the IHD, Paris, 2-14 September 1974, 42-53.
Jasechko, S., Kirchner, J.W., Welker, J.M., McDonnell, J.J. (2016): Substantial proportion of global streamflow less than three months old. Nature Geoscience, 9(2), 126-129.

Kirchner, J.W. (2016a): Aggregation in environmental systems-Part 1: Seasonal tracer cycles quantify young water fractions, but not mean transit times, in spatially heterogeneous catchments. Hydrol. Earth Syst. Sci., 20(1), 279-297. https://doi.org/10.5194/hess-20-2792016.

Kirchner, J.W. (2016b): Aggregation in environmental systems-Part 2: Catchment mean transit times and young water fractions under hydrologic nonstationarity. Hydrol. Earth Syst. Sci., 20(1), 299-328. https://doi.org/10.5194/ hess-20-299-2016.

Klaus, J. and McDonnell, J.J. (2013): Hydrograph separation using stable isotopes: Review and evaluation, Journal of Hydrology, 505, 47-64. https://doi.org/10.1016/j.jhydrol.2013.09.006.

Maier, F. (2017): Young water fraction in a highelevation catchment: temporal variability and relation to meteorological and glacio-hydrological proxies for climate change (Master thesis), Innsbruck.

McGuire, K.J. and McDonnell, J.J. (2006): A review and evaluation of catchment transit time 


\section{Originalarbeit}

modeling, Journal of Hydrology, 330, 543-563, https://doi.org/10.1016/j.jhydrol.2006.04.020.

Rixen, C., Teich, M., Lardelli, C., Gallati, D. Pohl, M., Puetz, M. and Bebi, P. (2011): Winter tourism and climate change in the Alps: an assessment of resource consumption, snow reliability, and future snowmaking potential. $M t$. Res. Dev. 31, 229-236.

Schaefli, B., Hingray, B., and Musy, A. (2007) Climate change and hydropower production in the Swiss Alps: quantification of potential impacts and related modelling uncertainties, Hydrol. Earth Syst. Sci., 11, 1191-1205, https:// doi.org/10.5194/hess-11-1191-2007.

Schmieder, J., Hanzer, F., Marke, T., Garvelmann, J., Warscher, M., Kunstmann, H., and Strasser, U. (2016): The importance of snowmelt spatiotemporal variability for isotope based hydrograph separation in a high-ele- vation catchment, Hydrol. Earth Syst. Sci., 20, 5015-5033, https://doi.org/10.5194/hess-205015-2016.

Schmieder, J., Marke, T., Garvelmann, J. and Strasser, U. (2018): Spatio-temporal tracer variability in the glacier melt end-member-How does it affect hydrograph separation results? Hydrological Processes.

Strasser, U. (2008): Modelling of the mountain snow cover in the Berchtesgaden National Park. Berchtesgaden National Park, Research Report (Vol. 55), ISBN 978-3-922325-62-8
Strasser, U, Marke, T, Braun, L, Escher-Vetter, H., Juen, I., Kuhn, M., Maussion, F., Mayer C., Nicholson, L., Niedertscheider, K., Sailer, R., Stötter, J., Weber, M. and Kaser, G. (2018): The Rofental: a high Alpine research basin (1890-3770 m a.s.l.) in the Ötztal Alps (Austria) with over 150 years of hydrometeorological and glaciological observations. Earth Syst. Sci. Data, 10(1), 151-171. https://doi.org/10.5194/ essd-10-151-2018. 